\title{
FAR FIELD BOUNDARY CONDITION FOR CONVECTION DIFFUSION EQUATION AT ZERO VISCOSITY LIMIT
}

\author{
BY \\ JIAN-GUO LIU (Department of Mathematics, Fudan University, Shanghai, P. R. China and \\ Institute for Physical Science and Technology and Department of Mathematics, University of \\ Maryland, College Park, MD 20742)
}

AND

WEN-QING XU (Department of Mathematics and Statistics, California State University, Long Beach, CA 90840)

\begin{abstract}
In this paper, we give a systematic study of the boundary layer behavior for linear convection-diffusion equation in the zero viscosity limit. We analyze the boundary layer structures in the viscous solution and derive the boundary condition satisfied by the viscosity limit as a solution of the inviscid equation. The results confirm that the Neumann type of far-field boundary condition is preferred in the outlet and characteristic boundary condition. Under some appropriate regularity and compatibility conditions on the initial and boundary data, we obtain optimal error estimates between the full viscous solution and the inviscid solution with suitable boundary layer corrections. These results hold in arbitrary space dimensions and similar statements also hold for the strip problem.

This model well describes the behavior at the far-field for many physical and engineering systems such as fluid dynamical equation and electro-magnetic equation. The results obtained here should provide some theoretical guidance for designing effective far-field boundary conditions.
\end{abstract}

1. Introduction. The purpose of this study is two-fold: understanding the boundary layer behavior in the zero viscosity limit for convection diffusion equations, and providing some theoretical guidance for designing effective far-field conditions.

At far-field, most physical quantities tend to constants. Hence the underlying physical systems can be well approximated by systems of linear convection diffusion equations with constant coefficients. In the simplest case, we have the following scalar convection diffusion equation

$$
\partial_{t} u^{\varepsilon}+\lambda \cdot \nabla u^{\varepsilon}=\varepsilon \Delta u^{\varepsilon}
$$

Received March 15, 2001.

2000 Mathematics Subject Classification. Primary 35K50, 35L50.

The research was supported in part by NSF grant DMS-0107218.

E-mail address: jliu@math.umd.edu

E-mail address: wxu@csulb.edu 
with initial data

$$
u^{\varepsilon}(x, 0)=u_{0}(x)
$$

where $x=\left(x_{1}, \cdots, x_{n}\right) \in \mathbf{R}^{n}, n \geq 1$ is the space dimension, $\lambda=\left(\lambda_{1}, \cdots, \lambda_{n}\right) \in \mathbf{R}^{n}$, and $\varepsilon>0$ represents the total dissipation, from physical mechanism or numerical viscosity, and is usually very small.

Cutting off the far-field domain from the computational domain and imposing some far-field boundary condition is one common approach in handling the far-field in computation. (Other methods are reduction of the far-field to some boundary integral equation [6], infinite element method [21], etc.) The study of the effective far-field boundary condition has been an important subject in the computation of fluid dynamics and computation of electro-magnetic wave [1]. Most of the previous research is for the inviscid case $[1,2,4,7,11,16]$. The addition of the dissipative term to the system usually gives rise to additional boundary conditions which in turn causes the solution to develop a sharp transition layer near the boundary. In reality, no matter how small it is, the dissipative term is always there. In this paper, we consider general mixed boundary conditions and present a systematic study of the effect of the viscosity term and the farfield boundary condition, particularly, the boundary layer behavior in the zero viscosity limit. The goal is to compare the solution behavior under different boundary conditions and therefore provide some theoretical guidance for designing effective far-field boundary conditions for weakly dissipative systems. In particular, we recover the well-known advantage of Neumann type far-field boundary conditions for weakening the boundary layers. We note that the Dirichlet boundary condition case has previously been studied by several authors; see for example, $[9,10,3,5]$. In the case of fixed viscosity term or parabolic equation, we refer to $[11,17,18,13]$ for the consistence and stability analysis on the numerical boundary conditions. We also refer to $[15,19,20]$ for the study of the boundary layer problem in the kinetic equations and relaxation systems.

The most natural boundary condition for the far-ficld is given by

$$
A u^{\varepsilon}\left(0, x^{\prime}, t\right)+B \partial_{x_{1}} u^{\varepsilon}\left(0, x^{\prime}, t\right)=b\left(x^{\prime}, t\right)
$$

where $x^{\prime}=\left(x_{2}, \cdots, x_{n}\right) \in \mathbf{R}^{n-1}$ and $A, B$ are constants with $(A, B) \neq(0,0)$. It is clear that for fixed $\varepsilon>0$, (1)-(3) consists of a well-posed parabolic initial boundary value problem in the quarter space $x_{1}, t>0$.

When the total dissipation approaches zero, formally one obtains from (1) the following inviscid equation

$$
\partial_{t} u+\lambda \cdot \nabla u=0
$$

Therefore one expects that in the zero viscosity limit, one should recover a solution of the inviscid equation (4) satisfying

$$
u(x, 0)=u_{0}(x) .
$$

This should be so, for instance, when (1) is used as a numerical approximation to (4) and $\varepsilon$ is the corresponding numerical viscosity.

For the Cauchy problem, this can be easily verified by either the energy method or the Fourier transform. Moreover, the convergence is uniform for any smooth initial data. The last statement follows from the next Lemma and the Sobolev embedding theorem. 
LEMma 1.1 (Cauchy problem). Let $u_{0}(x) \in L^{2}\left(\mathbf{R}^{n}\right)$. Let $u^{\varepsilon}$ and $u$ be the solution of (1) $-(2)$ and (4) $-(5)$ respectively. Then we have for any $\alpha>0$,

$$
\int_{0}^{\infty} \int_{\mathbf{R}^{n}}\left|u^{\varepsilon}(x, t)-u(x, t)\right|^{2} e^{-2 \alpha t} d x d t \rightarrow 0 \quad \text { as } \varepsilon \rightarrow 0 .
$$

If we assume further $u_{0} \in H^{2}\left(\mathbf{R}^{n}\right)$, then we also have

$$
\int_{0}^{\infty} \int_{\mathbf{R}^{n}}\left|u^{\varepsilon}(x, t)-u(x, t)\right|^{2} e^{-2 \alpha t} d x d t \leq O(1) \varepsilon^{2}\left\|u_{0}\right\|_{H^{2}\left(\mathbf{R}^{n}\right)}^{2}
$$

where the constant $O(1)$ is independent of $\varepsilon$ and $u_{0}(x)$ (but may depend on $\alpha$ ).

However, when the initial boundary value problem is concerned, the question becomes more subtle. This is the main subject of study in this paper. Most notably, we observe that there is a possible disagreement in the number of boundary conditions required for the parabolic viscous problem and the hyperbolic inviscid problem. For example, even for $n=1$, it is clear that in the cases $\lambda<0$ and $\lambda=0$, the solution of (4) is completely determined by the initial data $u(x, 0)=u_{0}(x), x \geq 0$ and no boundary condition is needed. Then the question arises: how does the boundary condition (3) affect the convergence of (1) to (4) in the zero viscosity limit?

It turns out that the desired convergence $u^{\varepsilon}(x, t) \rightarrow u(x, t)$ as $\varepsilon \rightarrow 0$ still holds, for example, in the same sense of (6). However, near the boundary, there is now a sharp transition which is known as a boundary layer. The effect of the boundary layer is limited to a narrow range near the boundary and its structure and magnitude depend on the specific form of the boundary condition (3) and whether $\lambda_{1}<0$ or $\lambda_{1}=0$. However, it is through this boundary layer that the discrepancy between the boundary conditions is resolved. Intuitively, one can think of the viscous solution of (1)-(3) as a superposition of the inviscid solution of (4)-(5) and the corresponding boundary layer and their higher order corrections. More precisely, we have the following main theorem of this paper.

Theorem 1.2 (Zero viscosity limit). Let $u^{\varepsilon}$ be the solution of $(1)-(3)$ and $b\left(x^{\prime}, t\right) \in$ $L^{2}\left(\mathbf{R}^{n-1} \times \mathbf{R}^{+}\right)$and $u_{0}(x) \in H^{1}\left(\mathbf{R}_{+}^{n}\right)$ with $u_{0}\left(0, x^{\prime}\right)=0$. Then there exists a unique limit $u(x, t)$ such that for all $\alpha>0$ sufficiently large,

$$
\int_{0}^{\infty} \int_{\mathbf{R}_{+}^{n}}\left|u^{\varepsilon}(x, t)-u(x, t)\right|^{2} e^{-2 \alpha t} d x d t \rightarrow 0 \quad \text { as } \varepsilon \rightarrow 0 .
$$

The zero viscosity limit $u(x, t)$ satisfies (4) and (5). In the case $\lambda_{1}>0$, it also satisfies the boundary condition $A u\left(0, x^{\prime}, t\right)+B \partial_{x_{1}} u\left(0, x^{\prime}, t\right)=b\left(x^{\prime}, t\right)$.

Under higher order regularity and compatibility conditions on the initial and boundary data, we can also obtain the following optimal error estimates.

Theorem 1.3 (Optimal error estimates). Assume further $b\left(x^{\prime}, t\right) \in H^{2}\left(\mathbf{R}^{n-1} \times \mathbf{R}^{+}\right)$, $u_{0}(x) \in H^{2}\left(\mathbf{R}_{+}^{n}\right)$ with $b\left(x^{\prime}, 0\right)=\partial_{t} b\left(x^{\prime}, 0\right)=0, u_{0}\left(0, x^{\prime}\right)=\partial_{x_{1}} u_{0}\left(0, x^{\prime}\right)=0$; then we 
have

$$
\begin{aligned}
& \int_{0}^{\infty} \int_{\mathbf{R}_{+}^{n}}\left|u^{\varepsilon}(x, t)-u(x, t)\right|^{2} e^{-2 \alpha t} d \cdot r d t
\end{aligned}
$$

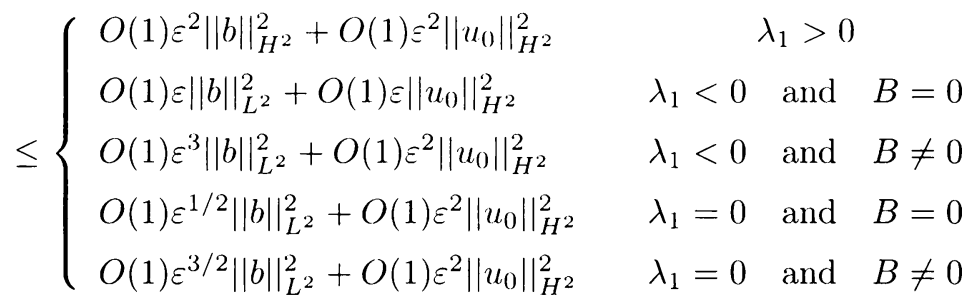

By further subtracting the boundary layer from the viscous solution, we can improve the above convergence estimates and hence rigorously justify the boundary layer structures in the cases $\lambda_{1}<0$ and $\lambda_{1}=0$.

TheOREm 1.4 (Boundary layer estimates). Let $b\left(x^{\prime} . t\right) \in H^{1}\left(\mathbf{R}^{n-1} \times \mathbf{R}^{+}\right), u_{0}(x) \in$ $H^{5}\left(\mathbf{R}_{+}^{n}\right)$ with $b\left(x^{\prime}, 0\right)=0, u_{0}\left(0, x^{\prime}\right)=\partial_{x_{1}} u_{0}\left(0, x^{\prime}\right)=\partial_{x_{1}}^{2} u_{0}\left(0, x^{\prime}\right)=0$; then there exists a boundary layer $u^{b . l} \cdot(x, t)$ in the cases $\lambda_{1}<0$ and $\lambda_{1}=0$ (with $u^{b . l .}(x, t)=0$ if $B \neq 0$ ) such that

$$
\begin{aligned}
& \int_{0}^{\infty} \int_{\mathbf{R}_{+}^{n}}\left|u^{\varepsilon}(x, t)-u(x, t)-u^{b . l .}(x, t)\right|^{2} e^{-2 \alpha t} d x d t \\
& \quad \leq \begin{cases}O(1) \varepsilon^{3}\|b\|_{H^{1}}^{2}+O(1) \varepsilon^{2}\left\|u_{0}\right\|_{H^{5}}^{2} & \lambda_{1}<0 \\
O(1) \varepsilon^{3 / 2}\|b\|_{H^{1}}^{2}+O(1) \varepsilon^{2}\left\|u_{0}\right\|_{H^{2}}^{2} & \lambda_{1}=0 .\end{cases}
\end{aligned}
$$

We note that the sign of $\lambda_{1}$ in the above theorems determines the main structure of the boundary layer. When $\lambda_{1}>0\left(\lambda_{1}<0\right)$, the problem corresponds to the inlet (outlet) problem. When $\lambda_{1}=0$, the problem is usually referred to as a characteristic boundary value problem and the boundary layer has a scaling $\varepsilon^{1 / 2}$ similar to the Prandtl scaling for a no-slip boundary condition. We also note that when $B \neq 0$ in the outlet and characteristic boundary condition, because of an otherwise large gradient term $\partial_{x_{1}} u^{b . l .}\left(0, x^{\prime}, t\right)$ in the boundary condition, the boundary layer is suppressed to the next order $O(\varepsilon)$ and therefore much weaker than the case of $B=0$. This explains why the Neumann boundary condition is preferred in these cases. Additionally, one may further suppress the boundary layer to order $O\left(\varepsilon^{2}\right)$ by choosing

$$
b\left(x^{\prime}, t\right)=A u_{0}\left(-\lambda_{1} t, x^{\prime}-\lambda^{\prime} t\right)+B \partial_{x_{1}} u_{0}\left(-\lambda_{1} t, x^{\prime}-\lambda^{\prime} t\right)
$$

with $B \neq 0$.

It should also be clear that under higher regularity and compatibility assumptions on the initial and boundary data, the above convergence rates can be further improved by including higher order corrections of the inviscid and boundary layer solutions. Furthermore, similar convergence estimates also hold for higher order Sobolev norms. Accordingly, point-wise convergence estimates with optimal convergence rates can be obtained by using Sobolev inequalities.

The proof of the above theorems will be carried out in the following sections. The plan is as follows. The one-dimensional case is considered first and the IBVP (1)-(3) is 
explicitly solved in Sec. 2 by Fourier-Laplace transform. The solution is then compared with its leading asymptotic behavior formally derived through matched asymptotic expansions. The desired estimates are then proved by using Parseval's identity and some detailed algebraic asymptotic analysis. The case with zero initial data is easier and is considered first in Sec. 3 while the more difficult nonzero initial data case is considered in Sec. 4. Next the multi-dimensional case is proved in Sec. 5. Finally we make some concluding remarks in Sec. 6 .

2. Solution by Laplace Transform. In this section we consider the simplest onedimensional case $(n=1)$ and solve the IBVP (1)-(3) (in the quarter plane $x, t>0$ ) explicitly by the method of Laplace transform $[12,8]$. Let $\xi=\alpha+i \beta$ with $\alpha=\operatorname{Re} \xi>0$ and define

$$
\tilde{u}^{\varepsilon}(x, \xi)=\int_{0}^{\infty} e^{-\xi t} u^{\varepsilon}(x, t) d t .
$$

We obtain the following ODE for $\tilde{u}^{\varepsilon}(x, \xi)$ :

$$
\varepsilon \partial_{x}^{2} \tilde{u}^{\varepsilon}-\lambda \partial_{x} \tilde{u}^{\varepsilon}-\xi \tilde{u}^{\varepsilon}=-u_{0}(x) .
$$

The initial data $u^{\varepsilon}(x, 0)=u_{0}(x)$ enters into the above ODE as an inhomogeneous term since

$$
\widetilde{\partial_{t} u^{\varepsilon}}=\xi \tilde{u}^{\varepsilon}(x, \xi)-u^{\varepsilon}(x, 0)=\xi \tilde{u}^{\varepsilon}(x, \xi)-u_{0}(x) .
$$

On the other hand, the boundary condition becomes

$$
A \tilde{u}^{\varepsilon}(0, \xi)+B \partial_{x} \tilde{u}^{\varepsilon}(0, \xi)=\tilde{b}(\xi)
$$

where $\tilde{b}(\xi)$ is the Laplace transform of the boundary data $b(t)$ given by

$$
\tilde{b}(\xi)=\int_{0}^{\infty} e^{-\xi t} b(t) d t .
$$

For convenience, we introduce

$$
\tilde{v}^{\varepsilon}(x, \xi)=\partial_{x} \tilde{u}^{\varepsilon}(x, \xi)
$$

and rewrite the above ODE (13) in the following first order system form:

$$
\partial_{x}\left(\begin{array}{c}
\tilde{u}^{\varepsilon} \\
\tilde{v}^{\varepsilon}
\end{array}\right)=M\left(\begin{array}{c}
\tilde{u}^{\varepsilon} \\
\tilde{v}^{\varepsilon}
\end{array}\right)+\left(\begin{array}{c}
0 \\
-\varepsilon^{-1} u_{0}(x)
\end{array}\right)
$$

where

$$
M=M(\xi, \varepsilon)=\left(\begin{array}{cc}
0 & 1 \\
\xi / \varepsilon & \lambda / \varepsilon
\end{array}\right) .
$$

The eigenvalues of the matrix $M$ can be easily found to be

$$
\omega_{ \pm}=\omega_{ \pm}(\xi, \varepsilon)=\frac{\lambda \pm \sqrt{\lambda^{2}+4 \varepsilon \xi}}{2 \varepsilon}
$$

with corresponding eigenvectors $r_{ \pm}=\left(1, \omega_{ \pm}\right)^{T}$.

By using the elementary inequality

$$
\operatorname{Re} \sqrt{\lambda^{2}+4 \varepsilon \xi} \geq \sqrt{\lambda^{2}+4 \varepsilon \alpha},
$$


it is easy to see that

$$
\operatorname{Re} \omega_{+}(\xi, \varepsilon)>0, \quad \operatorname{Re} \omega_{-}(\xi, \varepsilon)<0
$$

for all $\xi$ with $\alpha=\operatorname{Re} \xi>0$, and

$$
\operatorname{Re} \omega_{+}(\xi, \varepsilon) \rightarrow \infty, \quad-\operatorname{Re} \omega_{-}(\xi, \varepsilon) \rightarrow-\infty, \quad \text { as } \alpha \rightarrow \infty
$$

uniformly in $\beta$ and $\varepsilon$. Furthermore, we have

Lemma 2.1 (Properties of the eigenvalues $\omega_{ \pm}(\xi, \varepsilon)$ ). For $\alpha>0$ sufficiently large, there exists a constant $O(1)$ independent of $\beta$ and $\varepsilon$ such that

$$
\begin{aligned}
& \frac{1}{\operatorname{Re} \omega_{+}(\xi, \varepsilon)} \leq\left\{\begin{array}{cc}
O(1) \varepsilon & \lambda>0 \\
O(1) \sqrt{\varepsilon} & \lambda=0 \\
O(1) & \lambda<0
\end{array}\right. \\
& \frac{1}{-\operatorname{Re} \omega_{-}(\xi, \varepsilon)} \leq\left\{\begin{array}{cc}
O(1) & \lambda>0 \\
O(1) \sqrt{\varepsilon} & \lambda=0 \\
O(1) \varepsilon & \lambda<0
\end{array}\right. \\
& \omega_{+}(\xi, \varepsilon)=\left\{\begin{array}{cc}
\lambda / \varepsilon+O(1) \xi & \lambda>0 \\
\sqrt{\xi / \varepsilon} & \lambda=0 \\
-\xi / \lambda+O(1) \varepsilon \xi^{2} & \lambda<0
\end{array}\right. \\
& \omega_{-}(\xi, \varepsilon)=\left\{\begin{array}{cc}
-\xi / \lambda+O(1) \varepsilon \xi^{2} & \lambda>0 \\
-\sqrt{\xi / \varepsilon} & \lambda=0 \\
\lambda / \varepsilon+O(1) \xi & \lambda<0 .
\end{array}\right.
\end{aligned}
$$

Note that (22) in particular implies that $\omega_{+} \neq \omega_{-}$for all $\operatorname{Re} \xi>0$ and $\varepsilon>0$. Let $\Phi_{+}$ and $\Phi_{-}$be the projections onto $r_{+}$and $r_{-}$respectively, i.e.,

$$
\begin{aligned}
& \Phi_{+}=\frac{1}{\omega_{+}-\omega_{-}}\left(\begin{array}{c}
1 \\
\omega_{+}
\end{array}\right)\left(-\omega_{-}, 1\right), \\
& \Phi_{-}=\frac{1}{\omega_{-}-\omega_{+}}\left(\begin{array}{c}
1 \\
\omega_{-}
\end{array}\right)\left(-\omega_{+}, 1\right) ;
\end{aligned}
$$

then we have

$$
e^{M x}=e^{\omega_{+} x} \Phi_{+}+e^{\omega_{-} x} \Phi_{-}
$$

and hence the following solution representation of (18)

$$
\begin{aligned}
\left(\begin{array}{c}
\tilde{u}^{\varepsilon}(x, \xi) \\
\tilde{v}^{\varepsilon}(x, \xi)
\end{array}\right)= & e^{\omega_{+} x} \Phi_{+}\left(\left(\begin{array}{c}
\tilde{u}^{\varepsilon}(0, \xi) \\
\tilde{v}^{\varepsilon}(0, \xi)
\end{array}\right)+\int_{0}^{x} e^{-\omega_{+} y}\left(\begin{array}{c}
0 \\
-\varepsilon^{-1} u_{0}(y)
\end{array}\right) d y\right) \\
& +e^{\omega_{-} x} \Phi_{-}\left(\left(\begin{array}{c}
\tilde{u}^{\varepsilon}(0, \xi) \\
\tilde{v}^{\varepsilon}(0, \xi)
\end{array}\right)+\int_{0}^{x} e^{-\omega_{-} y}\left(\begin{array}{c}
0 \\
-\varepsilon^{-1} u_{0}(y)
\end{array}\right) d y\right)
\end{aligned}
$$


where the boundary data $\left(\tilde{u}^{\varepsilon}(0, \xi), \tilde{v}^{\varepsilon}(0, \xi)\right)$ remains to be determined.

The boundary data $\left(\tilde{u}^{\varepsilon}(0, \xi), \tilde{v}^{\varepsilon}(0, \xi)\right)$ clearly has to satisfy (15). However, in order to determine a unique solution $\left(\tilde{u}^{\varepsilon}(\cdot, \xi), \tilde{v}^{\varepsilon}(\cdot, \xi)\right) \in L^{2}\left(\mathbf{R}^{+}\right)$, one also needs the following boundary condition at $x=\infty$

$$
\tilde{u}^{\varepsilon}(+\infty, \xi)=0, \quad \tilde{v}^{\varepsilon}(+\infty, \xi)=0
$$

which, by (31) and Lemma 2.1, implies

$$
-\omega_{-} \tilde{u}^{\varepsilon}(0, \xi)+\tilde{v}^{\varepsilon}(0, \xi)=\varepsilon^{-1} \int_{0}^{\infty} e^{-\omega_{+} y} u_{0}(y) d y .
$$

This together with (15) yields

$$
\begin{aligned}
\left(\begin{array}{c}
\tilde{u}^{\varepsilon}(0, \xi) \\
\tilde{v}^{\varepsilon}(0, \xi)
\end{array}\right)= & \frac{\tilde{b}(\xi)}{A+B \omega_{-}}\left(\begin{array}{c}
1 \\
\omega_{-}
\end{array}\right) \\
& +\frac{1}{\varepsilon\left(A+B \omega_{-}\right)} \int_{0}^{\infty} e^{-\omega_{+} y} u_{0}(y) d y\left(\begin{array}{c}
-B \\
A
\end{array}\right) .
\end{aligned}
$$

Note that by choosing $\alpha>0$ large enough, we have from Lemma 2.1

$$
\left|A+B \omega_{-}(\xi, \varepsilon)\right| \geq C
$$

for some constant $C>0$ independent of $0<\varepsilon \leq \varepsilon_{0}$ and $\beta \in \mathbf{R}$. This guarantees the solvability of the boundary data $\left(\tilde{u}^{\varepsilon}(0, \xi), \tilde{v}^{\varepsilon}(0, \xi)\right)$ from (15) and (33).

With $(34)$, the desired solution $\tilde{u}^{\varepsilon}(x, \xi)$ can now be conveniently represented as

$$
\tilde{u}^{\varepsilon}(x, \xi)=\tilde{u}_{\mathrm{I}}^{\varepsilon}(x, \xi)+\tilde{u}_{\mathrm{II}}^{\varepsilon}(x, \xi)+\tilde{u}_{\mathrm{II}}^{\varepsilon}(x, \xi)
$$

where

$$
\begin{gathered}
\tilde{u}_{\mathrm{I}}^{\varepsilon}(x, \xi)=\frac{\tilde{b}(\xi)}{A+B \omega_{-}} e^{\omega_{-} x} \\
\tilde{u}_{\mathbb{I}}^{\varepsilon}(x, \xi)=\frac{1}{\varepsilon\left(\omega_{+}-\omega_{-}\right)} e^{\omega_{-} x} \int_{0}^{x} e^{-\omega_{-} y} u_{0}(y) d y \\
\\
+\frac{1}{\varepsilon\left(\omega_{+}-\omega_{-}\right)} e^{\omega_{+} x} \int_{x}^{\infty} e^{-\omega_{+} y} u_{0}(y) d y
\end{gathered}
$$

and

$$
\tilde{u}_{\mathrm{II}}^{\varepsilon}(x, \xi)=-\frac{1}{\varepsilon\left(\omega_{+}-\omega_{-}\right)} \frac{A+B \omega_{+}}{A+B \omega_{-}} e^{\omega_{-} x} \int_{0}^{\infty} e^{-\omega_{+} y} u_{0}(y) d y .
$$

The above solution decomposition allows us to greatly simplify our original IBVP (1)(3). It is easy to see that the first part $\tilde{u}_{\mathrm{I}}^{\varepsilon}(x, \xi)$ only involves the boundary condition and is the Laplace transform of the solution $u_{\mathrm{I}}^{\varepsilon}(x, t)$ to the following IBVP with homogeneous initial data

$$
\begin{gathered}
\partial_{t} u_{\mathrm{I}}^{\varepsilon}+\lambda \partial_{x} u_{\mathrm{I}}^{\varepsilon}=\varepsilon \partial_{x}^{2} u_{\mathrm{I}}^{\varepsilon}, \\
u_{\mathrm{I}}^{\varepsilon}(x, 0)=0, \\
A u_{\mathrm{I}}^{\varepsilon}(0, t)+B \partial_{x} u_{\mathrm{I}}^{\varepsilon}(0, t)=b(t) .
\end{gathered}
$$

Next, we notice that the second part $\tilde{u}_{\mathbb{I}}^{\varepsilon}(x, \xi)$, on the other hand, only involves the initial data and is independent of the boundary condition. Therefore $\tilde{u}_{\mathbb{I I}}^{\varepsilon}(x, \xi)$, or more 
precisely, its inverse Laplace transform $u_{\mathbb{I}}^{\varepsilon}(x, t)$, should solve a related Cauchy problem. This is indeed the case and one can show that $u_{\mathrm{II}}^{\varepsilon}(x, t)$ is exactly the solution of the following extended Cauchy problem

$$
\begin{gathered}
\partial_{t} u_{\mathrm{II}}^{\varepsilon}+\lambda \partial_{x} u_{\mathrm{II}}^{\varepsilon}=\varepsilon \partial_{x}^{2} u_{\mathrm{II}}^{\varepsilon} \\
u_{\mathrm{II}}^{\varepsilon}(x, 0)=\left\{\begin{array}{cc}
u_{0}(x) & x \geq 0 \\
0 & x<0 .
\end{array}\right.
\end{gathered}
$$

Finally, by linearity, we conclude that the last part $\tilde{u}_{\mathrm{m}}^{\varepsilon}(x, \xi)$ corresponds to the Laplace transform of the solution $u_{\mathrm{II}}^{\varepsilon}(x, t)$ to the following adjusted IBVP

$$
\begin{gathered}
\partial_{t} u_{\mathrm{II}}^{\varepsilon}+\lambda \partial_{x} u_{\mathrm{II}}^{\varepsilon}=\varepsilon \partial_{x}^{2} u_{\mathrm{II}}^{\varepsilon} \\
u_{\mathrm{III}}^{\varepsilon}(x, 0)=0 \\
A u_{\mathrm{II}}^{\varepsilon}(0, t)+B \partial_{x} u_{\mathrm{III}}^{\varepsilon}(0, t)=-\left(A u_{\mathrm{II}}^{\varepsilon}(0, t)+B \partial_{x} u_{\mathrm{II}}^{\varepsilon}(0, t)\right) .
\end{gathered}
$$

We will study each of these three problems separately. $u_{\mathrm{I}}^{\varepsilon}$ has a simple representation in terms of Laplace transform and can be estimated easily by using Parseval's relation and an asymptotic analysis. The estimate for $u_{\mathrm{II}}^{\varepsilon}$ follows from the results on Cauchy problem (Lemma 1.1). The main difficulty is with the last part $u_{\text {III }}^{\varepsilon}$.

3. Convergence Analysis: Zero Initial Data Case. In this section, we assume $u_{0}(x) \equiv 0$ and study the IBVP (40) first. For convenience, we drop the subscripts and still use $u^{\varepsilon}$ and $\tilde{u}^{\varepsilon}$ instead of $u_{\mathrm{I}}^{\varepsilon}$ and $\tilde{u}_{\mathrm{I}}^{\varepsilon}$.

From the previous section, we have

$$
\tilde{u}^{\varepsilon}(x, \xi)=\frac{\tilde{b}(\xi)}{A+B \omega_{-}(\xi, \varepsilon)} e^{\omega_{-}(\xi, \varepsilon) x} .
$$

Using Parseval's identity $[12,8]$ and Lemma 2.1, we obtain immediately

$$
\begin{aligned}
& \int_{0}^{\infty} \int_{0}^{\infty} e^{-2 \alpha t}\left|u^{\varepsilon}(x, t)\right|^{2} d x d t \\
& \quad=\frac{1}{2 \pi} \int_{0}^{\infty} \int_{-\infty}^{\infty}\left|\tilde{u}^{\varepsilon}(x, \alpha+i \beta)\right|^{2} d x d \beta \\
& \quad \leq O(1) \sup _{\beta} \frac{1}{-\operatorname{Re} \omega-(\xi, \varepsilon)} \int_{-\infty}^{\infty}|\tilde{b}(\alpha+i \beta)|^{2} d \beta \\
& \quad \leq\left\{\begin{array}{cc}
O(1) \int_{0}^{\infty} e^{-2 \alpha t}|b(t)|^{2} d t & \lambda>0 \\
O(1) \varepsilon \int_{0}^{\infty} e^{-2 \alpha t}|b(t)|^{2} d t & \lambda<0 \\
O(1) \sqrt{\varepsilon} \int_{0}^{\infty} e^{-2 \alpha t}|b(t)|^{2} d t & \lambda=0 .
\end{array}\right.
\end{aligned}
$$

By Lemma 2.1 , it is also clear that for $B \neq 0$, slightly stronger results can be obtained in the cases $\lambda<0$ and $\lambda=0$ :

$$
\begin{aligned}
& \int_{0}^{\infty} \int_{0}^{\infty} e^{-2 \alpha t}\left|u^{\varepsilon}(x, t)\right|^{2} d x d t \\
& \quad \leq\left\{\begin{array}{cl}
O(1) \varepsilon^{3} \int_{0}^{\infty} e^{-2 \alpha t}|b(t)|^{2} d t & \lambda<0 \\
O(1) \varepsilon^{3 / 2} \int_{0}^{\infty} e^{-2 \alpha t}|b(t)|^{2} d t & \lambda=0
\end{array} \quad(B \neq 0) .\right.
\end{aligned}
$$


The above estimates establish the asymptotic convergence of the solution $u^{\varepsilon}(x, t)$ to the trivial inviscid limit $u(x, t) \equiv 0$ as $\varepsilon \rightarrow 0$ in the cases $\lambda<0$ and $\lambda=0$. The convergence rates are optimal due to the presence of boundary layers. The last estimates in (45) show that the leading boundary layer also vanishes in these two cases with any non-Dirichlet boundary condition, i.e., $B \neq 0$. More insight will be given in the next subsection by using formal matched asymptotic expansions.

3.1. Matched asymptotic expansions. In order to identify the limiting behavior and the corresponding boundary layer structures in the solution $u^{\varepsilon}(x, t)$ of the viscous IBVP (40) as $\varepsilon \rightarrow 0$, we assume the following uniformly valid asymptotic expansions in terms of $\varepsilon$ :

$$
u^{\varepsilon}(x, t)=\left(u(x, t)+u^{b . l .}(x / \varepsilon, t)\right)+\varepsilon\left(u_{1}(x, t)+u_{1}^{b . l .}(x / \varepsilon, t)\right)+\cdots
$$

with the localized boundary layers $u^{b . l .}(y, t)$ and $u_{1}^{b . l .}(y, t)$ decaying sufficiently fast as $y=x / \varepsilon \rightarrow+\infty$.

Plugging the above expansions into Eq. (40) and matching the orders of $\varepsilon$, at the leading order, we obtain the following equations:

$$
\begin{gathered}
\partial_{t} u+\lambda \partial_{x} u=0 \\
\partial_{y}^{2} u^{b . l .}-\lambda \partial_{y} u^{b . l .}=0 .
\end{gathered}
$$

Note that the leading order Hilbert solution $u(x, t)$ satisfies the inviscid equation (4). Clearly the initial condition for $u(x, 0)$ should be

$$
u(x, 0)=u_{0}(x) \equiv 0 .
$$

No boundary condition is needed in the cases $\lambda<0$ and $\lambda=0$. Hence the solution is completely determined by the initial data

$$
u(x, t) \equiv 0 \quad(\lambda \leq 0) .
$$

This is clearly consistent with the convergence results in (44) and (45).

In the case $\lambda>0$, a boundary condition for $u(0, t)$ is needed. This will be determined shortly by a matching of the boundary condition for (40).

Next we look at the leading order boundary layer $u^{\text {b.l. }}(y, t)$. We only have to solve the linear $\mathrm{ODE}(47)_{2}$ subject to the decay requirement $u^{b . l .}(y, t) \rightarrow 0$ as $y \rightarrow+\infty$. The solution can be immediately obtained by direct integration:

$$
u^{b . l .}(y, t)=\left\{\begin{array}{cc}
0 & \lambda>0 \\
u^{b . l .}(0, t) e^{\lambda y} & \lambda<0 .
\end{array}\right.
$$

Therefore, no boundary layer develops in the case $\lambda>0$. In the case $\lambda<0$, the boundary layer $u^{b . l .}(y, t)$ decays exponentially fast as $y \rightarrow+\infty$.

We now turn to determining the boundary condition for $u(0, t)$ (when $\lambda>0$ ) and $u^{b . l .}(0, t)$ (when $\lambda<0$ ). This is easily achieved by matching the boundary conditions. Plugging (46) into the boundary condition in (40) and separating different orders of $\varepsilon$, we arrive at

$$
\begin{gathered}
B \partial_{y} u^{b . l \cdot}(0, t)=0 \\
A\left(u(0, t)+u^{b . l \cdot}(0, t)\right)+B\left(\partial_{x} u(0, t)+\partial_{y} u_{1}^{b . l \cdot}(0, t)\right)=b(t) .
\end{gathered}
$$


Therefore, for $\lambda>0$, the matching of boundary condition in (51) yields

$$
A u(0, t)+B \partial_{x} u(0, t)=b(t)
$$

and for $\lambda<0$, we have

$$
u^{b . l .}(0, t)=\left\{\begin{array}{cl}
b(t) / A & \text { if } B=0 \\
0 & \text { if } B \neq 0 .
\end{array}\right.
$$

The solution for $u(x, t)$ and $u^{b . l .}(y, t)$ follows then easily.

We now consider the remaining case of $\lambda=0$. It is easy to see that the above expansion procedure fails in this case and the matching of boundary conditions as in (51) becomes impossible unless in the trivial case $b(t) \equiv 0$. This is because for $\lambda=0$, the boundary $x=0$ becomes uniformly characteristic and the boundary layer is now of a different type and actually lives on a much larger scale of $\sqrt{\varepsilon}$ near $x=0$. The previous expansion (46) is therefore unable to capture such boundary layer behavior in the present case. In view of the convergence estimate in (44) and analogous results in the relaxation case [19], we now choose the following asymptotic expansions:

$$
u^{\varepsilon}(x, t)=u(x, t)+u^{b . l .}(y, t)+\sqrt{\varepsilon} u_{1}^{b . l .}(y, t)+O(\varepsilon)
$$

where $y=x / \sqrt{\varepsilon}$ and $u^{b . l .}(y, t), u_{1}^{b . l .}(y, t) \rightarrow 0$ as $y \rightarrow+\infty$.

Substituting (54) into (40) and matching the orders of $\varepsilon$, we obtain the same inviscid equation

$$
\partial_{t} u+\lambda \partial_{x} u=0
$$

for the leading order Hilbert solution $u(x, t)$. It is clear that

$$
u(x, t) \equiv 0
$$

with the choice of homogeneous initial data $u(x, 0)=u_{0}(x) \equiv 0$.

However, the leading order boundary layer $u^{b . l}(y, t)$ is now governed by the following diffusion equation

$$
\partial_{t} u^{b . l .}=\partial_{y}^{2} u^{b . l}
$$

This is quite different from the $\mathrm{ODE}(47)_{2}$ in the case $\lambda \neq 0$. To determine the solution uniquely, we need both initial data $u^{b . l .}(y, 0)$ and boundary data $u^{b . l .}(0, t)$. Again the initial data should be

$$
u^{b . l .}(y, 0) \equiv 0
$$

while the necessary boundary data $u^{b . l}(0, t)$ can be determined from the same matching of boundary condition as in (51) which again gives

$$
u^{b . l .}(0, t)=\left\{\begin{array}{cl}
b(t) / A & \text { if } B=0 \\
0 & \text { if } B \neq 0 .
\end{array}\right.
$$

The boundary layer $u^{b . l}$. $(y, t)$ can then be obtained by solving the parabolic IBVP (57)(59). 
Thus we have determined all the leading order Hilbert and boundary layer solutions in all the cases $\lambda>0, \lambda<0$, and $\lambda=0$. For later convenience, we represent them in terms of Laplace transform and summarize our formal expansion results as follows:

$$
\begin{gathered}
\tilde{u}(x, \xi)=\left\{\begin{array}{cc}
\frac{\tilde{b}(\xi)}{A-B \xi / \lambda} e^{-\xi x / \lambda} & \lambda>0 \\
0 & \lambda \leq 0
\end{array}\right. \\
\tilde{u}^{b . l .}(x, \xi)=\left\{\begin{array}{ccc}
0 & \lambda>0 & \text { or } \quad B \neq 0 \\
\frac{1}{A} \tilde{b}(\xi) e^{\lambda x / \varepsilon} & \lambda<0 & \text { and } \quad B=0 \\
\frac{1}{A} \tilde{b}(\xi) e^{-x \sqrt{\xi} / \sqrt{\varepsilon}} & \lambda=0 & \text { and } B=0 .
\end{array}\right.
\end{gathered}
$$

3.2. Convergence proof. We now rigorously justify the formal convergence results obtained in the previous subsection. We start with the convergence proof

$$
\int_{0}^{\infty} \int_{0}^{\infty} e^{-2 \alpha t}\left|u^{\varepsilon}(x, t)-u(x, t)\right|^{2} d x d t \rightarrow 0 \quad \text { as } \quad \varepsilon \rightarrow 0,
$$

where $\alpha>0$ is the same (large enough) constant we have chosen and fixed before.

Indeed, in the cases $\lambda<0$ and $\lambda=0$, stronger convergence results than the above have been obtained; see (44) and (45). We only have to prove the above estimate (62) for the case $\lambda>0$. Note that in this case, we have

$$
\tilde{u}^{\varepsilon}(x, \xi)=\frac{\tilde{b}(\xi)}{A+B \omega_{-}(\xi, \varepsilon)} e^{\omega_{-}(\xi, \varepsilon) x}
$$

and

$$
\tilde{u}(x, \xi)=\frac{\tilde{b}(\xi)}{A-B \xi / \lambda} e^{-\xi x / \lambda}
$$

The point-wise convergence of $\tilde{u}^{\varepsilon}(x, \xi) \rightarrow \tilde{u}(x, \xi)$ holds obviously since in this case $\omega_{-}(\xi, \varepsilon) \rightarrow-\xi / \lambda$ as $\varepsilon \rightarrow 0$. On the other hand, we have by direct integration

$$
\int_{0}^{\infty} \int_{-\infty}^{\infty}\left(\left|\tilde{u}^{\varepsilon}(x, \xi)\right|^{2}+|\tilde{u}(x, \xi)|^{2}\right) d x d \beta \leq O(1) \int_{-\infty}^{\infty}|\tilde{b}(\xi)|^{2} d \beta .
$$

Therefore, by Lebesgue's dominated convergence theorem, we have

$$
\int_{0}^{\infty} \int_{-\infty}^{\infty}\left|\tilde{u}^{\varepsilon}(x, \xi)-\tilde{u}(x, \xi)\right|^{2} d x d \beta \rightarrow 0 \quad \text { as } \quad \varepsilon \rightarrow 0
$$

which, by Parseval's identity, implies the convergence in (62).

An optimal convergence rate can be obtained if we further assume $b(t) \in H^{2}\left(\mathbf{R}^{+}\right)$and $b(t)$ satisfies the compatibility condition $b(0)=b^{\prime}(0)=0$. First, we write

$$
\begin{aligned}
\tilde{u}^{\varepsilon}(x, \xi)-\tilde{u}(x, \xi)= & \frac{\tilde{b}(\xi)}{A+B \omega_{-}(\xi, \varepsilon)}\left(e^{\omega_{-}(\xi, \varepsilon) x}-e^{-\xi x / \lambda}\right) \\
& +\left(\frac{1}{A+B \omega_{-}(\xi, \varepsilon)}-\frac{1}{A-B \xi / \lambda}\right) \tilde{b}(\xi) e^{-\xi x / \lambda}
\end{aligned}
$$


Next using Lemma 2.1 (with $\alpha$ large enough), we have

$$
\left|\frac{1}{A+B \omega_{-}(\xi, \varepsilon)}-\frac{1}{A-B \xi / \lambda)}\right| \leq O(1)\left|\omega_{-}(\xi, \varepsilon)+\xi / \lambda\right| \leq O(1) \varepsilon|\xi|^{2}
$$

and

$$
\int_{0}^{\infty}\left|e^{\omega_{-}(\xi . \varepsilon) x}-e^{-\xi x / \lambda}\right|^{2} d x \leq O(1)\left|\omega_{-}(\xi, \varepsilon)+\xi / \lambda\right|^{2} \leq O(1) \varepsilon^{2}|\xi|^{4} .
$$

Combining the above, we now obtain

$$
\begin{aligned}
\int_{0}^{\infty} \int_{-\infty}^{\infty}\left|\tilde{u}^{\varepsilon}(x, \xi)-\tilde{u}(x, \xi)\right|^{2} d x d \beta & \leq O(1) \int_{-\infty}^{\infty} \varepsilon^{2}|\xi|^{4}|\tilde{b}(\xi)|^{2} d \beta \\
& \leq O(1) \varepsilon^{2} \int_{0}^{\infty} e^{-2 \alpha t}\left|b^{\prime \prime}(t)\right|^{2} d t .
\end{aligned}
$$

Next we remark that the above analysis can also be used to rigorously justify the boundary layer structures given $(61)$. With $u_{0}(x) \equiv 0$, it is clear that the leading order Hilbert solution, i.e., the inviscid limit $u(x, t)$, vanishes identically in the cases $\lambda<0$ and $\lambda=0$. In fact, the same conclusion also holds for higher order Hilbert solutions. Therefore, in such cases, the IBVP (40) is all about boundary layers. This is best illustrated in the extreme case of $\lambda=0$ with Dirichlet boundary condition, i.e., $B=0$. It is remarkable that in this case the whole viscous solution $u^{\varepsilon}(x, t)$ coincides exactly with the leading order boundary layer $u^{b . l .}(x / \sqrt{\varepsilon}, t)$. For all other boundary conditions, that is, $B \neq 0$, both the estimate (45) and the formal expansion show that the boundary layers are only present at higher orders, and are therefore much weaker. The same is true when $\lambda<0$, though the boundary layers now live on a different scale; see again the estimate in (45).

4. Convergence Analysis: Nonzero Initial Data Case. We now turn to the nonzero initial data case and consider the remaining two parts $u_{\mathrm{II}}^{\varepsilon}(x, t)$ and $u_{\mathrm{II}}^{\varepsilon}(x, t)$ in the solution representation (36). Without confusion, we assume $b(t) \equiv 0$ in this section. Then it is clear that

$$
u^{\varepsilon}(x, t)=u_{\mathrm{II}}^{\varepsilon}(x, t)+u_{\mathrm{II}}^{\varepsilon}(x, t)
$$

solves the following IBVP

$$
\begin{gathered}
\partial_{t} u^{\varepsilon}+\lambda \partial_{x} u^{\varepsilon}=\varepsilon \partial_{x}^{2} u^{\varepsilon} \\
u^{\varepsilon}(x, 0)=u_{0}(x) \\
A u^{\varepsilon}(0, t)+B \partial_{x} u^{\varepsilon}(0, t)=0 .
\end{gathered}
$$

We will prove similar convergence results as in the last section. In particular, we show that there exists a unique inviscid limit $u(x, t)$ with $e^{-\alpha t} u(x, t) \in L^{2}\left(\mathbf{R}^{+} \times \mathbf{R}^{+}\right)$such that

$$
\int_{0}^{\infty} \int_{0}^{\infty} e^{-2 \alpha t}\left|u^{\varepsilon}(x, t)-u(x, t)\right|^{2} d x d t \rightarrow 0 \quad \text { as } \varepsilon \rightarrow 0
$$


This inviscid limit $u(x, t)$, together with any possible boundary layers, again can be formally derived by the same matched asymptotic expansions as in the last section. For (72), assuming compatibility conditions $u_{0}(0)=u_{0}^{\prime}(0)=0$, we now get

$$
\begin{gathered}
u(x, t)=\left\{\begin{array}{cc}
u_{0}(x-\lambda t) & x \geq \lambda t \\
0 & x<\lambda t
\end{array}, \quad u^{b . l .}(x, t)=0 \quad(\lambda>0)\right. \\
u(x, t)=u_{0}(x-\lambda t), \quad u^{b . l .}(x, t)=\left\{\begin{array}{cc}
-u_{0}(-\lambda t) e^{\lambda x / \varepsilon} & B=0 \\
0 & B \neq 0
\end{array} \quad(\lambda<0)\right.
\end{gathered}
$$

and

$$
u(x, t)=u_{0}(x), \quad u^{b . l .}(x, t)=0 \quad(\lambda=0) .
$$

We shall first look at $u_{\mathrm{II}}^{\varepsilon}(x, t)$. Since $u_{\mathrm{I}}^{\varepsilon}(x, t)$ solves the extended Cauchy problem (41), from Lemma 1.1, we obtain immediately

$$
\int_{0}^{\infty} \int_{0}^{\infty} e^{-2 \alpha t}\left|u_{\Pi}^{\varepsilon}(x, t)-u_{\mathbb{I}}(x, t)\right|^{2} d x d t \leq O(1) \varepsilon^{2} \int_{0}^{\infty}\left|u_{0}^{\prime \prime}(x)\right|^{2} d x
$$

where $u_{\mathrm{II}}(x, t)$ is the inviscid limit for the extended Cauchy problem (41), that is,

$$
u_{\mathbb{I}}(x, t)=\left\{\begin{array}{cc}
u_{0}(x-\lambda t) & x \geq \lambda t \\
0 & x<\lambda t .
\end{array}\right.
$$

It is interesting to note that when restricted to the first quadrant $x, t>0$, the two inviscid limits always coincide, i.e.,

$$
u_{\mathrm{II}}(x, t) \equiv u(x, t) \quad \text { for } \quad x \geq 0 .
$$

This clearly suggests that $u_{\mathrm{II}}^{\varepsilon}(x, t) \rightarrow u_{\mathrm{III}}(x, t) \equiv 0$ in the following sense:

$$
\int_{0}^{\infty} \int_{0}^{\infty} e^{-2 \alpha t}\left|u_{\mathrm{III}}^{\varepsilon}(x, t)\right|^{2} d x d t \rightarrow 0 \quad \text { as } \quad \varepsilon \rightarrow 0
$$

or equivalently,

$$
\int_{0}^{\infty} \int_{-\infty}^{\infty}\left|\tilde{u}_{\mathrm{III}}^{\varepsilon}(x, \xi)\right|^{2} d x d \beta \rightarrow 0 \quad \text { as } \quad \varepsilon \rightarrow 0
$$

Recall that

$$
\tilde{u}_{\mathrm{II}}^{\varepsilon}(x, \xi)=-\frac{1}{\varepsilon\left(\omega_{+}-\omega_{-}\right)} \frac{A+B \omega_{+}}{A+B \omega_{-}} e^{\omega_{-} x} \int_{0}^{\infty} e^{-\omega_{+} y} u_{0}(y) d y .
$$

Using Cauchy-Schwarz inequality, we have

$$
\left|\int_{0}^{\infty} e^{-\omega_{+} y} u_{0}(y) d y\right|^{2} \leq \frac{1}{2 \operatorname{Re} \omega_{+}} \int_{0}^{\infty}\left|u_{0}(y)\right|^{2} d y
$$

Next, by direct calculation, we have

$$
\begin{aligned}
& \int_{-\infty}^{\infty} \frac{1}{\left|\varepsilon\left(\omega_{+}-\omega_{-}\right)\right|^{2}} \frac{1}{-\operatorname{Re} \omega_{-}} \frac{1}{\operatorname{Re} \omega_{+}} d \beta \\
& \quad \leq O(1) \int_{-\infty}^{\infty} \frac{\varepsilon}{\lambda^{2}+\varepsilon|\xi|^{2}} d \beta \leq\left\{\begin{array}{cc}
O(1) & \lambda=0 \\
O(1) \sqrt{\varepsilon} & \lambda \neq 0 .
\end{array}\right.
\end{aligned}
$$


Therefore for Dirichlet boundary condition $(B=0)$, we obtain immediately,

$$
\begin{aligned}
& \int_{0}^{\infty} \int_{-\infty}^{\infty}\left|\tilde{u}_{\mathrm{II}}^{\varepsilon}(x, \xi)\right|^{2} d x d \beta \\
& \quad \leq\left\{\begin{array}{cc}
O(1) \int_{0}^{\infty}\left|u_{0}(y)\right|^{2} d y & \lambda=0 \\
O(1) \sqrt{\varepsilon} \int_{0}^{\infty}\left|u_{0}(y)\right|^{2} d y & \lambda \neq 0
\end{array} \quad(B=0) .\right.
\end{aligned}
$$

Next, by taking $\alpha=\operatorname{Re} \xi$ large enough, we have for non-Dirichlet boundary conditions $(B \neq 0)$

$$
\left|\frac{A+B \omega_{+}}{A+B \omega_{-}}\right| \leq O(1)\left|\frac{\omega_{+}}{\omega_{-}}\right| .
$$

On the other hand, a simple integration by parts yields (assuming $u_{0} \in H^{1}$ and $u_{0}(0)=0$ )

$$
\int_{0}^{\infty} e^{-\omega_{+} y} u_{0}(y) d y=\frac{1}{\omega_{+}} \int_{0}^{\infty} e^{-\omega_{+} y} u_{0}^{\prime}(y) d y .
$$

Note that by Lemma 2.1, the integrated factor $1 / \omega_{+}$gives us a convergence factor of $\varepsilon$ and $\varepsilon^{1 / 2}$ in the cases $\lambda>0$ and $\lambda=0$ respectively. Therefore, by the same analysis as above, we get for $B \neq 0$,

$$
\begin{aligned}
& \int_{0}^{\infty} \int_{-\infty}^{\infty}\left|\tilde{u}_{\mathrm{II}}^{\varepsilon}(x, \xi)\right|^{2} d x d \beta \\
& \quad \leq O(1) \int_{0}^{\infty} \int_{-\infty}^{\infty}\left|\omega_{-}\right|^{-2}\left|\frac{1}{\varepsilon\left(\omega_{+}-\omega_{-}\right)} e^{\omega_{-} x} \int_{0}^{\infty} e^{-\omega_{+} y} u_{0}^{\prime}(y) d y\right|^{2} d x d \beta \\
& \quad \leq\left\{\begin{array}{ll}
O(1) \varepsilon^{5 / 2} \int_{0}^{\infty}\left|u_{0}^{\prime}(y)\right|^{2} d y & \lambda<0 \\
O(1) \varepsilon \int_{0}^{\infty}\left|u_{0}^{\prime}(y)\right|^{2} d y & \lambda=0 \\
O(1) \varepsilon^{1 / 2} \int_{0}^{\infty}\left|u_{0}^{\prime}(y)\right|^{2} d y & \lambda>0
\end{array} \quad(B \neq 0) .\right.
\end{aligned}
$$

The above estimate also holds for Dirichlet boundary condition $(B=0)$ when $\lambda>0$ or $\lambda=0$. We further remark that by a repeated application of integration by parts as in (87), we can get (assuming enough regularity and compatibility conditions)

$$
\begin{aligned}
& \int_{0}^{\infty} \int_{-\infty}^{\infty}\left|\tilde{u}_{\mathrm{II}}^{\varepsilon}(x, \xi)\right|^{2} d x d \beta \\
& \quad \leq \begin{cases}O(1) \varepsilon^{n} \int_{0}^{\infty}\left|u_{0}^{(n)}(y)\right|^{2} d y & \lambda=0 \\
O(1) \varepsilon^{2 n-3 / 2} \int_{0}^{\infty}\left|u_{0}^{(n)}(y)\right|^{2} d y & \lambda>0\end{cases}
\end{aligned}
$$

In the case $\lambda<0$, due to the presence of boundary layers (see (75)), the boundary effect arising from the initial data is no longer arbitrarily small. However, for Dirichlet boundary condition $(B=0)$, the boundary layer appears right at the leading order; for non-Dirichlet boundary conditions $(B \neq 0)$, the boundary layer is much weaker and only 
appears at order $\varepsilon$. This is already reflected in (85) and (88):

$$
\begin{aligned}
& \int_{0}^{\infty} \int_{-\infty}^{\infty}\left|\tilde{u}_{\mathrm{II}}^{\varepsilon}(x, \xi)\right|^{2} d x d \beta \\
& \quad \leq\left\{\begin{array}{ll}
O(1) \varepsilon^{1 / 2} \int_{0}^{\infty}\left|u_{0}(y)\right|^{2} d y & B=0 \\
O(1) \varepsilon^{5 / 2} \int_{0}^{\infty}\left|u_{0}^{\prime}(y)\right|^{2} d y & B \neq 0 .
\end{array} \quad(\lambda<0)\right.
\end{aligned}
$$

REMARK. Using a technique in [19], we can improve the above convergence result and obtain the following optimal convergence rates in the case $\lambda<0$ :

$$
\begin{aligned}
& \int_{0}^{\infty} \int_{-\infty}^{\infty}\left|\tilde{u}_{\mathrm{III}}^{\varepsilon}(x, \xi)\right|^{2} d x d \beta \\
& \quad \leq\left\{\begin{array}{ll}
O(1) \varepsilon \int_{0}^{\infty}\left|u_{0}(y)\right|^{2} d y & B=0 \\
O(1) \varepsilon^{3} \int_{0}^{\infty}\left|u_{0}^{\prime}(y)\right|^{2} d y & B \neq 0 .
\end{array} \quad(\lambda<0)\right.
\end{aligned}
$$

The proof proceeds as follows. First, by applying the $L^{2}$ energy method to IBVP (72) with Neumann boundary condition $\partial_{x} u^{\varepsilon}(0, t)=0$, we can obtain the following weighted $L^{2}$ estimate (uniformly in $\varepsilon$ ):

$$
\begin{aligned}
& \int_{0}^{\infty} \int_{0}^{\infty} e^{-2 \alpha t}\left|u^{\varepsilon}(x, t)\right|^{2} d x d t+\int_{0}^{\infty} e^{-2 \alpha t}\left|u^{\varepsilon}(0, t)\right|^{2} d t \\
& \quad \leq O(1) \int_{0}^{\infty}\left|u_{0}(x)\right|^{2} d x .
\end{aligned}
$$

Next we note that the boundary data $u^{\varepsilon}(0, t)$ has the following Laplace transform representation

$$
\tilde{u}^{\varepsilon}(0, \xi)=-\frac{1}{\varepsilon \omega_{-}} \int_{0}^{\infty} e^{-\omega_{+} y} u_{0}(y) d y \quad(A=0) .
$$

Therefore the boundary estimate, in particular, implies that

$$
\int_{-\infty}^{\infty}\left|\frac{1}{\varepsilon \omega_{-}} \int_{0}^{\infty} e^{-\omega_{+} y} u_{0}(y) d y\right|^{2} d \beta \leq O(1) \int_{0}^{\infty}\left|u_{0}(y)\right|^{2} d y \quad(\lambda<0) .
$$

We can now finish the proof of (91). For $B=0$, we have immediately,

$$
\int_{0}^{\infty} \int_{-\infty}^{\infty}\left|\tilde{u}_{\mathrm{III}}^{\varepsilon}(x, \xi)\right|^{2} d x d \beta \leq O(1) \varepsilon \int_{0}^{\infty}\left|u_{0}(y)\right|^{2} d y
$$

For $B \neq 0$, we only have to use an integration by parts as in (87),

$$
\begin{aligned}
& \int_{0}^{\infty} \int_{-\infty}^{\infty}\left|\tilde{u}_{\mathrm{II}}^{\varepsilon}(x, \xi)\right|^{2} d x d \beta \\
& \quad \leq O(1) \varepsilon^{3} \int_{-\infty}^{\infty}\left|\frac{1}{\varepsilon \omega_{-}} \int_{0}^{\infty} e^{-\omega_{+} y} u_{0}^{\prime}(y) d y\right|^{2} d \beta \\
& \quad \leq O(1) \varepsilon^{3} \int_{0}^{\infty}\left|u_{0}^{\prime}(y)\right|^{2} d y
\end{aligned}
$$


We now finish the proof of Theorem 1.4 by showing the following boundary layer estimate in the case of Dirichlet boundary condition $(\lambda<0$ and $B=0)$ :

$$
\int_{0}^{\infty} \int_{-\infty}^{\infty}\left|\tilde{u}_{\mathrm{II}}^{\varepsilon}(x, \xi)-\tilde{u}^{b . l .}(x, \xi)\right|^{2} d x d \beta \leq O(1) \varepsilon^{3} \int_{0}^{\infty}\left|u_{0}^{\prime \prime \prime}(x)\right|^{2} d x .
$$

Note that in this case, we have

$$
\tilde{u}_{\mathrm{II}}^{\varepsilon}(x, \xi)=-\frac{1}{\varepsilon\left(\omega_{+}-\omega_{-}\right)} e^{\omega_{-} x} \int_{0}^{\infty} e^{-\omega_{+} y} u_{0}(y) d y .
$$

On the other hand, from (75), it follows

$$
\tilde{u}^{b . l .}(x, \xi)=\lambda^{-1} e^{\lambda x / \varepsilon} \int_{0}^{\infty} e^{\xi y / \lambda} u_{0}(y) d y .
$$

To prove (97), we first apply the same trick of integration by parts as in (87) to $\tilde{u}_{\mathrm{II}}^{\varepsilon}(x, \xi)$ and $\tilde{u}^{b . l .}(x, \xi)$ to obtain

$$
\begin{gathered}
\tilde{u}_{\mathrm{III}}^{\varepsilon}(x, \xi)=-\frac{1}{\varepsilon\left(\omega_{+}-\omega_{-}\right) \omega_{+}^{3}} e^{\omega_{-} x} \int_{0}^{\infty} e^{-\omega_{+} y} u_{0}^{\prime \prime \prime}(y) d y \\
\tilde{u}^{b . l .}(x, \xi)=-\lambda^{2} \xi^{-3} e^{\lambda x / \varepsilon} \int_{0}^{\infty} e^{\xi y / \lambda} u_{0}^{\prime \prime \prime}(y) d y
\end{gathered}
$$

and therefore

$$
\begin{aligned}
\tilde{u}_{\mathrm{III}}^{\varepsilon}(x, \xi)-\tilde{u}^{b . l \cdot}(x, \xi) & \left(\frac{\lambda^{2}}{\xi^{3}}-\frac{1}{\varepsilon\left(\omega_{+}-\omega_{-}\right) \omega_{+}^{3}}\right) e^{\omega_{-} x} \int_{0}^{\infty} e^{-\omega_{+} y} u_{0}^{\prime \prime \prime}(y) d y \\
& -\lambda^{2} \xi^{-3}\left(e^{\omega_{-} x}-e^{\lambda x / \varepsilon}\right) \int_{0}^{\infty} e^{-\omega_{+} y} u_{0}^{\prime \prime \prime}(y) d y \\
& -\lambda^{2} \xi^{-3} e^{\lambda x / \varepsilon} \int_{0}^{\infty}\left(e^{-\omega_{+} y}-e^{\xi y / \lambda}\right) u_{0}^{\prime \prime \prime}(y) d y .
\end{aligned}
$$

Next we note that by using

$$
\omega_{+} \omega_{-}=-\xi / \varepsilon, \quad 1 / \omega_{+}+1 / \omega_{-}=-\lambda / \xi,
$$

it can be easily checked that

$$
\frac{\lambda^{2}}{\xi^{3}}-\frac{1}{\varepsilon\left(\omega_{+}-\omega_{-}\right) \omega_{+}^{3}}=\frac{1}{\xi \omega_{-}}\left(\frac{1}{\omega_{+}-\omega_{-}}-\frac{\lambda}{\xi}\right)=O(1) \varepsilon \xi^{-1} .
$$

Furthermore, similar to (69), we have

$$
\int_{0}^{\infty}\left|e^{-\omega_{+} x}-e^{\xi y / \lambda}\right|^{2} d y \leq O(1)\left|\omega_{+}+\xi / \lambda\right|^{2} \leq O(1) \varepsilon^{2}|\xi|^{4} .
$$

With the above estimates, the desired boundary layer estimate (97) now follows easily from the same analysis as before.

This completes the proof of Theorems $1.2-1.4$ in the case $n=1$. 
5. Multi-dimensional IBVP. We now turn to the proof of Theorems $1.2-1.4$ in the multi-dimensional case. For simplicity, we only present the proof in the 2-D case. The analysis is based on an additional Fourier transform on the tangential space variable. The proof is similar to the 1-D case and easily extends to higher dimensions.

5.1. Solution by Fourier-Laplace transform. Consider now the $2-\mathrm{D}$ viscous IBVP in the quarter space $x \geq 0, y \in \mathbf{R}$, and $t \geq 0$ :

$$
\begin{gathered}
\partial_{t} u^{\varepsilon}+\lambda \partial_{x} u^{\varepsilon}+\mu \partial_{y} u^{\varepsilon}=\varepsilon \Delta u^{\varepsilon} \\
u^{\varepsilon}(x, y, 0)=u_{0}(x, y) \\
A u^{\varepsilon}(0, y, t)+B \partial_{x} u^{\varepsilon}(0, y, t)=b(y, t),
\end{gathered}
$$

where $\Delta$ is the 2-D Laplacian operator, $\lambda \in \mathbf{R}, \mu \in \mathbf{R}$, and $0<\varepsilon \leq \varepsilon_{0}<\infty$.

Denote $\tilde{u}^{\varepsilon}(x, l, \xi)$ the Fourier-Laplace transform of $u^{\varepsilon}(x, y, t)$ :

$$
\tilde{u}^{\varepsilon}(x, l, \xi)=\int_{0}^{\infty} \int_{-\infty}^{\infty} e^{-\xi t-i l y} u^{\varepsilon}(x, y, t) d y d t
$$

where $l \in \mathbf{R}, \xi=\alpha+i \beta$. Again we only consider $\alpha=\operatorname{Re} \xi>0$ large enough.

The IBVP (106) is now transformed into the following ODE (regarding $l$ and $\xi$ as parameters)

$$
\varepsilon \partial_{x}^{2} \tilde{u}^{\varepsilon}-\lambda \partial_{x} \tilde{u}^{\varepsilon}-\left(\xi+i l \mu+\varepsilon l^{2}\right) \tilde{u}^{\varepsilon}=-\hat{u}_{0}(x, l),
$$

where $\hat{u}_{0}(x, l)$ is the Fourier transform (in $y$ only) of the initial data $u_{0}(x, y)$ :

$$
\hat{u}_{0}(x, l)=\int_{-\infty}^{\infty} e^{-i l y} u_{0}(x, y) d y
$$

The boundary condition now becomes

$$
A \tilde{u}^{\varepsilon}(0, l, \xi)+B \partial_{x} \tilde{u}^{\varepsilon}(0, l, \xi)=\tilde{b}(l, \xi)
$$

where $\tilde{b}(l, \xi)$ is the Fourier-Laplace transform of the boundary data $b(y, t)$ :

$$
\tilde{b}(l, \xi)=\int_{0}^{\infty} \int_{-\infty}^{\infty} e^{-\xi t-i l y} b(y, t) d y d t .
$$

The ODE (108) can be rewritten in the following first order system form:

$$
\partial_{x}\left(\begin{array}{c}
\tilde{u}^{\varepsilon} \\
\tilde{v}^{\varepsilon}
\end{array}\right)=M\left(\begin{array}{c}
\tilde{u}^{\varepsilon} \\
\tilde{v}^{\varepsilon}
\end{array}\right)+\left(\begin{array}{c}
0 \\
-\varepsilon^{-1} \hat{u}_{0}(x, l)
\end{array}\right),
$$

where

$$
\tilde{v}^{\varepsilon}(x, l, \xi)=\partial_{x} \tilde{u}^{\varepsilon}(x, l, \xi)
$$

and the new matrix $M$ is now given by

$$
M=M(\xi, l, \varepsilon)=\left(\begin{array}{cc}
0 & 1 \\
\left(\xi+i l \mu+\varepsilon l^{2}\right) / \varepsilon & \lambda / \varepsilon
\end{array}\right) .
$$

Besides the boundary condition (110), or equivalently,

$$
A \tilde{u}^{\varepsilon}(0, l, \xi)+B \tilde{v}^{\varepsilon}(0, l, \xi)=\tilde{b}(l, \xi)
$$


the solution $\left(\tilde{u}^{\varepsilon}(x, l, \xi), \tilde{v}^{\varepsilon}(x, l, \xi)\right)$ has also to satisfy the following implicit boundary condition at $x=+\infty$ :

$$
\tilde{u}^{\varepsilon}(+\infty, l, \xi)=0, \quad \tilde{v}^{\varepsilon}(+\infty, l, \xi)=0 .
$$

The eigenvalues of the matrix $M=M(\xi, l, \varepsilon)$ are now given by

$$
\omega_{ \pm}=\omega_{ \pm}(\xi, l, \varepsilon)=\frac{\lambda \pm \sqrt{\lambda^{2}+4 \varepsilon\left(\xi+i l \mu+\varepsilon l^{2}\right)}}{2 \varepsilon}
$$

with corresponding eigenvectors $r_{ \pm}=\left(1, \omega_{ \pm}\right)^{T}$.

Similar to Lemma 2.1 , we have the following estimates for $\omega_{ \pm}(\xi, l, \varepsilon)$ :

LEMmA 5.1 (Properties of $\omega_{ \pm}(\xi, l, \varepsilon)$ ). The eigenvalues $\omega_{ \pm}(\xi, l, \varepsilon)$ satisfy the following uniform estimates:

$$
\begin{gathered}
\operatorname{Re} \omega_{+}(\xi, l, \varepsilon)>0, \quad \operatorname{Re} \omega_{-}(\xi, l, \varepsilon)<0 \text { for all } \operatorname{Re} \xi>0, l \in \mathbf{R}, \varepsilon>0 \\
\pm \operatorname{Re} \omega_{ \pm}(\xi, l, \varepsilon) \rightarrow \infty \text { as } \alpha \rightarrow \infty \text { (uniformly in } \beta, l, \text { and } \varepsilon \text { ) }
\end{gathered}
$$

The appropriate boundary data and hence the solution can now be uniquely determined by the same procedure as in Sec. 2. Again the solution $\tilde{u}^{\varepsilon}(x, l, \xi)$ can be decomposed into three parts:

$$
\tilde{u}^{\varepsilon}(x, l, \xi)=\tilde{u}_{\mathrm{I}}^{\varepsilon}(x, l, \xi)+\tilde{u}_{\mathrm{II}}^{\varepsilon}(x, l, \xi)+\tilde{u}_{\mathrm{II}}^{\varepsilon}(x, l, \xi)
$$

with

$$
\tilde{u}_{\mathrm{I}}^{\varepsilon}(x, l, \xi)=\frac{\tilde{b}(l, \xi)}{A+B \omega_{-}} e^{\omega_{-} x}
$$




$$
\begin{aligned}
\tilde{u}_{\mathrm{II}}^{\varepsilon}(x, l, \xi)= & \frac{1}{\varepsilon\left(\omega_{+}-\omega_{-}\right)} e^{\omega_{-} x} \int_{0}^{x} e^{-\omega_{-} \eta} \hat{u}_{0}(\eta, l) d \eta \\
& +\frac{1}{\varepsilon\left(\omega_{+}-\omega_{-}\right)} e^{\omega_{+} x} \int_{x}^{\infty} e^{-\omega_{+} \eta} \hat{u}_{0}(\eta, l) d \eta
\end{aligned}
$$

and

$$
\tilde{u}_{\mathrm{II}}^{\varepsilon}(x, l, \xi)=-\frac{1}{\varepsilon\left(\omega_{+}-\omega_{-}\right)} \frac{A+B \omega_{+}}{A+B \omega_{-}} e^{\omega_{-} x} \int_{0}^{\infty} e^{-\omega_{+} \eta} \hat{u}_{0}(\eta, l) d \eta
$$

where $\omega_{ \pm}=\omega_{ \pm}(\xi, l, \varepsilon)$. Note that similar to (35), for $\alpha>0$ sufficiently large, we have (see Lemma 5.1),

$$
\left|A+B \omega_{-}(\xi, l, \varepsilon)\right| \geq C
$$

for some positive constant $C>0$ independent of $0<\varepsilon \leq \varepsilon_{0}, l \in \mathbf{R}$, and $\beta \in \mathbf{R}$.

As before, the first part $\tilde{u}_{\mathrm{I}}^{\varepsilon}(x, l, \xi)$ corresponds to the Fourier-Laplace transform of the solution $u_{\mathrm{I}}^{\varepsilon}(x, y, t)$ to the following IBVP with homogeneous initial data

$$
\begin{gathered}
\partial_{t} u_{\mathrm{I}}^{\varepsilon}+\lambda \partial_{x} u_{\mathrm{I}}^{\varepsilon}+\mu \partial_{y} u_{\mathrm{I}}^{\varepsilon}=\varepsilon \Delta u_{\mathrm{I}}^{\varepsilon} \\
u_{\mathrm{I}}^{\varepsilon}(x, y, 0)=0 \\
A u_{\mathrm{I}}^{\varepsilon}(0, y, t)+B \partial_{x} u_{\mathrm{I}}^{\varepsilon}(0, y, t)=b(y, t)
\end{gathered}
$$

The second part $\tilde{u}_{\Pi}^{\varepsilon}(x, l, \xi)$ is the Fourier-Laplace transform of the solution $u_{\mathbb{I}}^{\varepsilon}(x, y, t)$ of the following extended Cauchy problem

$$
\begin{array}{r}
\partial_{t} u_{\Pi}^{\varepsilon}+\lambda \partial_{x} u_{\Pi}^{\varepsilon}+\mu \partial_{y} u_{\mathrm{II}}^{\varepsilon}=\varepsilon \Delta u_{\mathrm{II}}^{\varepsilon} \\
u_{\mathrm{I}}^{\varepsilon}(x, y, 0)=\left\{\begin{array}{cc}
u_{0}(x, y) & x \geq 0 \\
0 & x<0
\end{array}\right.
\end{array}
$$

And the last part $\tilde{u}_{\mathrm{II}}^{\varepsilon}(x, l, \xi)$ corresponds to the Fourier-Laplace transform of the solution $u_{\mathrm{II}}^{\varepsilon}(x, y, t)$ to the following adjusted IBVP

$$
\begin{gathered}
\partial_{t} u_{\mathrm{II}}^{\varepsilon}+\lambda \partial_{x} u_{\mathrm{II}}^{\varepsilon}+\mu \partial_{y} u_{\mathrm{II}}^{\varepsilon}=\varepsilon \Delta u_{\mathrm{II}}^{\varepsilon} \\
u_{\mathrm{II}}^{\varepsilon}(x, y, 0)=0 \\
A u_{\mathrm{III}}^{\varepsilon}(0, y, t)+B \partial_{x} u_{\mathrm{II}}^{\varepsilon}(0, y, t)=-\left(A u_{\mathrm{II}}^{\varepsilon}(0, y, t)+B \partial_{x} u_{\mathrm{II}}^{\varepsilon}(0, y, t)\right) .
\end{gathered}
$$

5.2. Convergence analysis: Zero initial data case. In this subsection, we assume $u_{0}(x, y) \equiv 0$ and study the IBVP (129) first. Again we drop the subscripts and use $u^{\varepsilon}$ and $\tilde{u}^{\varepsilon}$ instead of $u_{\mathrm{I}}^{\varepsilon}$ and $\tilde{u}_{\mathrm{I}}^{\varepsilon}$.

Similar to (43), we now have

$$
\tilde{u}^{\varepsilon}(x, l, \xi)=\frac{\tilde{b}(l, \xi)}{A+B \omega_{-}(\xi, l, \varepsilon)} e^{\omega_{-}(\xi, l, \varepsilon) x}
$$


Therefore from Lemma 5.1 and Parseval's identity, we get

$$
\begin{aligned}
& \int_{0}^{\infty} \int_{0}^{\infty} \int_{-\infty}^{\infty} e^{-2 \alpha t}\left|u^{\varepsilon}(x, y, t)\right|^{2} d x d y d t \\
& \quad=\frac{1}{(2 \pi)^{2}} \int_{0}^{\infty} \int_{-\infty}^{\infty} \int_{-\infty}^{\infty}\left|\tilde{u}^{\varepsilon}(x, l, \alpha+i \beta)\right|^{2} d x d l d \beta \\
& \quad \leq O(1) \sup _{\beta, l} \frac{1}{-\operatorname{Re} \omega-(\xi, l, \varepsilon)} \int_{-\infty}^{\infty} \int_{-\infty}^{\infty}|\tilde{b}(l, \alpha+i \beta)|^{2} d l d \beta \\
& \quad \leq\left\{\begin{array}{cc}
O(1) \int_{0}^{\infty} \int_{-\infty}^{\infty} e^{-2 \alpha t}|b(y, t)|^{2} d y d t & \lambda>0 \\
O(1) \varepsilon \int_{0}^{\infty} \int_{-\infty}^{\infty} e^{-2 \alpha t}|b(y, t)|^{2} d y d t & \lambda<0 \\
O(1) \sqrt{\varepsilon} \int_{0}^{\infty} \int_{-\infty}^{\infty} e^{-2 \alpha t}|b(y, t)|^{2} d y d t & \lambda=0 .
\end{array}\right.
\end{aligned}
$$

For $B \neq 0$, the following slightly stronger estimates also hold in the cases $\lambda<0$ and $\lambda=0$ :

$$
\begin{aligned}
& \int_{0}^{\infty} \int_{0}^{\infty} \int_{-\infty}^{\infty} e^{-2 \alpha t}\left|u^{\varepsilon}(x, y, t)\right|^{2} d x d y d t \\
& \quad \leq\left\{\begin{array}{cll}
O(1) \varepsilon^{3} \int_{0}^{\infty} \int_{-\infty}^{\infty} e^{-2 \alpha t}|b(y, t)|^{2} d y d t & \lambda<0 \\
O(1) \varepsilon^{3 / 2} \int_{0}^{\infty} \int_{-\infty}^{\infty} e^{-2 \alpha t}|b(y, t)|^{2} d y d t & \lambda=0
\end{array} \quad(B \neq 0) .\right.
\end{aligned}
$$

This shows the asymptotic convergence of $u^{\varepsilon}(x, y, t) \rightarrow u(x, y, t) \equiv 0$ as $\varepsilon \rightarrow 0$ in the cases $\lambda<0$ and $\lambda=0$. For $\lambda>0$, the inviscid limit $u(x, y, t)$ still exists, but is no longer identically zero.

We now apply a similar asymptotic expansion procedure to the IBVP (129). This will determine the inviscid limit $u(x, y, t)$ in the case $\lambda>0$ and will also give the boundary layers in the cases $\lambda<0$ and $\lambda=0$.

For $\lambda \neq 0$, the expansion takes the form

$$
\begin{aligned}
u^{\varepsilon}(x, y, t)= & \left(u(x, y, t)+u^{b . l .}(x / \varepsilon, y, t)\right) \\
& +\varepsilon\left(u_{1}(x, y, t)+u_{1}^{b . l .}(x / \varepsilon, y, t)\right)+\cdots
\end{aligned}
$$

where the boundary layers $u^{b . l .}(\eta, y, t)$ and $u_{1}^{b . l .}(\eta, y, t)$ are assumed to decay exponentially fast as $\eta=x / \varepsilon \rightarrow+\infty$.

The governing equations for $u(x, y, t), u^{b . l}(\eta, y, t)$, etc., can be obtained by substituting (135) into the equation (129) and matching the orders of $\varepsilon$. At the leading order, we get

$$
\partial_{t} u+\lambda \partial_{x} u+\mu \partial_{y} u=0
$$

and

$$
\partial_{\eta}^{2} u^{b . l .}=\lambda \partial_{\eta} u^{b . l .}
$$

It remains to determine the suitable initial and boundary conditions for $u(x, y, t)$ and $u^{b . l .}(\eta, y, t)$. The initial condition for $u(x, y, 0)$ should clearly be

$$
u(x, y, 0)=u_{0}(x, y) \equiv 0 \quad(x \geq 0),
$$


while the appropriate boundary condition for $u(0, y, t)$ (when $\lambda>0$ ) and $u^{b . l .}(0, y, t)$ (when $\lambda<0$ ) have to be determined by a similar matching of boundary conditions as in the $1-\mathrm{D}$ case (see Sec. 3 ) which now yields

$$
A u(0, y, t)+B \partial_{x} u(0, y, t)=b(y, t)
$$

and

$$
u^{b . l .}(0, y, t)=\left\{\begin{array}{cl}
b(y, t) / A & \text { if } B=0 \\
0 & \text { if } B \neq 0
\end{array} \quad(\lambda<0) .\right.
$$

The solutions to (136) and (137) (in Fourier-Laplace transform) are now given by

$$
\begin{aligned}
& \tilde{u}(x, l, \xi)=\left\{\begin{array}{cc}
\frac{\tilde{b}(l, \xi)}{A-B(\xi+i l \mu) / \lambda} e^{-(\xi+i l \mu) x / \lambda} & \lambda>0 \\
0 & \lambda<0
\end{array}\right.
\end{aligned}
$$

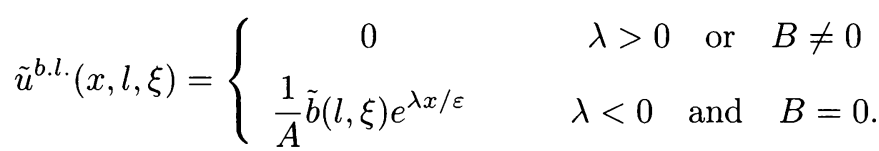

For $\lambda=0$, we have already seen that $u^{\varepsilon}(x, y, t) \rightarrow u(x, y, t) \equiv 0$ as $\varepsilon \rightarrow 0$. The boundary layers now live on a scale of $\sqrt{\varepsilon}$ near $x=0$ and the asymptotic expansion should have the form

$$
u^{\varepsilon}(x, y, t)=u(x, y, t)+u^{b . l .}(\eta, y, t)+\sqrt{\varepsilon} u_{1}^{b . l .}(\eta, y, t)+O(1) \varepsilon,
$$

where $\eta=x / \sqrt{\varepsilon}$ and $u^{b . l .}(\eta, y, t), u_{1}^{b . l .}(\eta, y, t) \rightarrow 0$ as $\eta \rightarrow+\infty$.

The leading order boundary layer $u^{\text {b.l. }}(\eta, y, t)$ now satisfies the following diffusion equation (with $y$ as a parameter):

$$
\partial_{t} u^{b . l .}=\partial_{\eta}^{2} u^{b . l .}
$$

On the other hand, a matching of boundary condition yields the same boundary data for $u^{b . l .}(0, y, t)$ as in the case $\lambda<0$ :

$$
u^{b . l .}(0, y, t)=\left\{\begin{array}{cl}
b(y, t) / A & \text { if } B=0 \\
0 & \text { if } B \neq 0 .
\end{array} \quad(\lambda=0)\right.
$$

The boundary layer $u^{b . l} \cdot(\eta, y, t)$ is now uniquely determined by (144) and (145) (and the initial condition $\left.u^{b . l .}(\eta, y, 0)=0\right)$. The solution is easily found in terms of FourierLaplace transform:

$$
\tilde{u}^{b . l .}(x, l, \xi)=\left\{\begin{array}{cl}
0 & B \neq 0 \\
\frac{1}{A} \tilde{b}(l, \xi) e^{-x \sqrt{\xi+i l \mu} / \sqrt{\varepsilon}} & B=0
\end{array} \quad(\lambda=0) .\right.
$$

The desired convergence of $\tilde{u}^{\varepsilon}(x, y, t) \rightarrow \tilde{u}(x, y, t)$ as $\varepsilon \rightarrow 0$ in the case of $\lambda>0$ can now be proved by a slight modification of the corresponding proof in Sec. 3.2 for the 1-D case. Similar to $(66)$, we now have

$$
\int_{0}^{\infty} \int_{-\infty}^{\infty} \int_{-\infty}^{\infty}\left|\tilde{u}^{\varepsilon}(x, l, \xi)-\tilde{u}(x, l, \xi)\right|^{2} d x d l d \beta \rightarrow 0 \quad \text { as } \quad \varepsilon \rightarrow 0 .
$$


Next using a similar decomposition for $\tilde{u}^{\varepsilon}(x, l, \xi)-\tilde{u}(x, l, \xi)$ as in $(67)$ and the following estimate (see Lemma 5.1)

$$
\left|\omega_{-}(\xi, l, \varepsilon)+(\xi+i l \mu) / \lambda\right| \leq O(1) \varepsilon\left(|\xi|^{2}+l^{2}\right) \quad(\lambda>0),
$$

we can obtain the following estimate with optimal convergence rate

$$
\begin{aligned}
& \int_{0}^{\infty} \int_{-\infty}^{\infty} \int_{-\infty}^{\infty}\left|\tilde{u}^{\varepsilon}(x, l, \xi)-\tilde{u}(x, l, \xi)\right|^{2} d x d l d \beta \\
& \quad \leq O(1) \int_{-\infty}^{\infty} \int_{-\infty}^{\infty}\left|\omega_{-}(\xi, l, \varepsilon)+(\xi+i l \mu) / \lambda\right|^{2}|\tilde{b}(l, \xi)|^{2} d l d \beta \\
& \quad \leq O(1) \int_{-\infty}^{\infty} \int_{-\infty}^{\infty} \varepsilon^{2}\left(|\xi|^{4}+l^{4}\right)|\tilde{b}(l, \xi)|^{2} d l d \beta \\
& \quad \leq O(1) \varepsilon^{2} \int_{0}^{\infty} \int_{-\infty}^{\infty} e^{-2 \alpha t}\left(\left|\partial_{y}^{2} b\right|^{2}+\left|\partial_{t}^{2} b\right|^{2}\right) d y d t \\
& \leq\left. O(1) \varepsilon^{2}|| b\right|_{H^{2}} ^{2}
\end{aligned}
$$

where we have assumed that $b(y, t) \in H^{2}\left(\mathbf{R} \times \mathbf{R}^{+}\right)$and $b(y, t)$ satisfies the compatibility condition $b(y, 0)=\partial_{t} b(y, 0)=0$.

The validity of the boundary layers in the cases $\lambda<0$ and $\lambda=0$ (see (142) or (146)) can be justified by similar estimates. Details are omitted.

5.3. Convergence analysis: Nonzero initial data case. We now look at the nonzero initial data effect in the IBVP (106) and consider the remaining two terms $u_{\mathrm{II}}^{\varepsilon}(x, y, t)$ and $u_{\mathrm{m}}^{\varepsilon}(x, y, t)$ in the solution representation (124). It is clear that

$$
u^{\varepsilon}(x, y, t)=u_{\mathrm{II}}^{\varepsilon}(x, y, t)+u_{\mathrm{II}}^{\varepsilon}(x, y, t)
$$

now solves the IBVP $(106)$ with $b(y, t) \equiv 0$.

The same matched asymptotic expansion procedures as in the last subsection can be used to find the formal asymptotics of the solution $u^{\varepsilon}(x, y, t)$ as $\varepsilon \rightarrow 0$. The inviscid limit $u(x, y, t)$ again satisfies

$$
\begin{gathered}
\partial_{t} u+\lambda \partial_{x} u+\mu \partial_{y} u=0 \\
u(x, y, 0)=u_{0}(x, y) \quad(x \geq 0)
\end{gathered}
$$

(supplemented with the boundary condition $u(0, y, t) \equiv 0$ in the case $\lambda>0$ ).

Similar to the corresponding 1-D case, the boundary layer vanishes in the case $\lambda=0$ (assuming $\left.u_{0}(0, y) \equiv 0\right)$; while in the case $\lambda<0$, we have

$$
u^{b . l .}(x / \varepsilon, y, t)=\left\{\begin{array}{cc}
-u_{0}(-\lambda t, y-\mu t) e^{\lambda x / \varepsilon} & B=0 \\
0 & B \neq 0
\end{array} \quad(\lambda<0) .\right.
$$

The inviscid limit $u(x, y, t)$ is associated with the extended Cauchy problem (130). It can be easily checked that the solution $u_{\mathbb{I}}(x, y, t)$ of the following inviscid Cauchy problem

$$
\begin{gathered}
\partial_{t} u_{\mathrm{II}}+\lambda \partial_{x} u_{\mathrm{II}}+\mu \partial_{y} u_{\mathrm{II}}=0 \\
u_{\mathrm{II}}(x, y, 0)=\left\{\begin{array}{cc}
u_{0}(x, y) & x \geq 0 \\
0 & x \leq 0
\end{array}\right.
\end{gathered}
$$


when restricted to $x>0$, gives exactly the desired inviscid limit $u(x, y, t)$ in all the cases $\lambda>0, \lambda<0$, and $\lambda=0$.

Therefore, as an immediate consequence of Lemma 1.1, we obtain

$$
\int_{0}^{\infty} \int_{0}^{\infty} \int_{-\infty}^{\infty} e^{-2 \alpha t}\left|u_{\mathrm{II}}^{\varepsilon}(x, y, t)-u(x, y, t)\right|^{2} d x d y d t \leq O(1) \varepsilon^{2}\left\|u_{0}\right\|_{H^{2}}^{2}
$$

To finish the proof of Theorems 1.2-1.4, it remains to show that $u_{\mathrm{m}}^{\varepsilon}(x, y, t) \rightarrow 0$ as $\varepsilon \rightarrow 0$ together with the appropriate convergence rates and a boundary layer estimate in the case $\lambda<0$.

Note that

$$
\tilde{u}_{\mathrm{III}}^{\varepsilon}(x, l, \xi)=-\frac{1}{\varepsilon\left(\omega_{+}-\omega_{-}\right)} \frac{A+B \omega_{+}}{A+B \omega_{-}} e^{\omega_{-} x} \int_{0}^{\infty} e^{-\omega_{+} \eta} \hat{u}_{0}(\eta, l) d \eta .
$$

Using Cauchy-Schwarz inequality, we get

$$
\left|\int_{0}^{\infty} e^{-\omega_{+} \eta} \hat{u}_{0}(\eta, l) d \eta\right|^{2} \leq \frac{1}{2 \operatorname{Re} \omega_{+}} \int_{0}^{\infty}\left|\hat{u}_{0}(\eta, l)\right|^{2} d \eta .
$$

Next, similar to (84), we have

$$
\begin{aligned}
& \int_{-\infty}^{\infty} \frac{1}{\left|\varepsilon\left(\omega_{+}-\omega_{-}\right)\right|^{2}} \frac{1}{-\operatorname{Re} \omega_{-}} \frac{1}{\operatorname{Re} \omega_{+}} d \beta \\
& \quad \leq O(1) \int_{-\infty}^{\infty} \frac{\varepsilon}{\lambda^{2}+\varepsilon|\xi+i l \mu|^{2}} d \beta \leq\left\{\begin{array}{cc}
O(1) & \lambda=0 \\
O(1) \sqrt{\varepsilon} & \lambda \neq 0 .
\end{array}\right.
\end{aligned}
$$

Furthermore, by Parseval's identity, we have

$$
\int_{0}^{\infty} \int_{-\infty}^{\infty}\left|\hat{u}_{0}(\eta, l)\right|^{2} d \eta d l=\frac{1}{2 \pi} \int_{0}^{\infty} \int_{-\infty}^{\infty}\left|u_{0}(\eta, y)\right|^{2} d \eta d y
$$

Therefore for Dirichlet boundary condition $(B=0)$, we obtain immediately,

$$
\begin{aligned}
& \int_{0}^{\infty} \int_{-\infty}^{\infty} \int_{-\infty}^{\infty}\left|\tilde{u}_{\mathrm{II}}^{\varepsilon}(x, l, \xi)\right|^{2} d x d l d \beta \\
& \quad \leq\left\{\begin{array}{cc}
O(1)\left\|u_{0}\right\|_{L^{2}}^{2} & \lambda=0 \\
O(1) \sqrt{\varepsilon}\left\|u_{0}\right\|_{L^{2}}^{2} & \lambda \neq 0
\end{array} \quad(B=0) .\right.
\end{aligned}
$$

This estimate, while crude, is essential in proving (through integration by part as in Sec. 4)

$$
\int_{0}^{\infty} \int_{-\infty}^{\infty} \int_{-\infty}^{\infty}\left|\tilde{u}_{\mathrm{II}}^{\varepsilon}(x, l, \xi)\right|^{2} d x d l d \beta \leq O(1) \varepsilon^{5 / 2}\left\|u_{0}\right\|_{H^{1}}^{2} \quad(\lambda<0, B \neq 0)
$$

for non-Dirichlet boundary conditions in the case $\lambda<0$ and

$$
\begin{aligned}
& \int_{0}^{\infty} \int_{-\infty}^{\infty} \int_{-\infty}^{\infty}\left|\tilde{u}_{\mathrm{II}}^{\varepsilon}(x, l, \xi)\right|^{2} d x d l d \beta \\
& \quad \leq\left\{\begin{array}{lll}
O(1) \varepsilon^{n} \int_{0}^{\infty} \int_{-\infty}^{\infty}\left|\partial_{x}^{n} u_{0}(x, y)\right|^{2} d x d y & \lambda=0 \\
O(1) \varepsilon^{2 n-3 / 2} \int_{0}^{\infty} \int_{-\infty}^{\infty}\left|\partial_{x}^{n} u_{0}(x, y)\right|^{2} d x d y & \lambda>0
\end{array} \quad(n \geq 1)\right.
\end{aligned}
$$

for both Dirichlet and non-Dirichlet boundary conditions in the cases $\lambda>0$ and $\lambda=0$. 
With the help of the weighted $L^{2}$ energy estimate (see Sec. 4), the estimates in the case $\lambda<0$ can be improved to vield optimal convergence rates:

$$
\begin{aligned}
& \int_{0}^{\infty} \int_{-\infty}^{\infty} \int_{-\infty}^{\infty}\left|\tilde{u}_{\mathrm{II}}^{\varepsilon}(x, l, \xi)\right|^{2} d x d l d \beta \\
& \quad \leq\left\{\begin{array}{ll}
O(1) \varepsilon\left\|u_{0}\right\|_{L^{2}}^{2} & B=0 \\
O(1) \varepsilon^{3}\left\|u_{0}\right\|_{H^{1}}^{2} & B \neq 0
\end{array} \quad(\lambda<0) .\right.
\end{aligned}
$$

Finally the desired boundary layer estimate in the case of Dirichlet boundary condition $(B=0$ and $\lambda<0)$

$$
\int_{0}^{\infty} \int_{-\infty}^{\infty} \int_{-\infty}^{\infty}\left|\tilde{u}_{\mathrm{III}}^{\varepsilon}(x, l, \xi)-\tilde{u}^{b . l .}(x, l, \xi)\right|^{2} d x d l d \beta \leq O(1) \varepsilon^{3}\left\|u_{0}\right\|_{H^{5}}^{2}
$$

follows from a similar treatment as in the 1-D case. Details are omitted.

\section{Further Remarks.}

6.1. Strip Problem. The analysis in the previous sections can also be applied to study the convergence of (1) to (4) and the associated boundary layer behaviors in more general cases. Here we briefly discuss the following strip problem

$$
\begin{gathered}
\partial_{t} u^{\varepsilon}+\lambda \cdot \nabla u^{\varepsilon}=\varepsilon \Delta u^{\varepsilon} \\
u^{\varepsilon}(x, 0)=u_{0}(x) \\
A_{0} u^{\varepsilon}\left(0, x^{\prime}, t\right)+B_{0} \partial_{x_{1}} u^{\varepsilon}\left(0, x^{\prime}, t\right)=b_{0}\left(x^{\prime}, t\right) \\
A_{1} u^{\varepsilon}\left(1, x^{\prime}, t\right)+B_{1} \partial_{x_{1}} u^{\varepsilon}\left(1, x^{\prime}, t\right)=b_{1}\left(x^{\prime}, t\right)
\end{gathered}
$$

where $0 \leq x_{1} \leq 1$. Consider, for example, $\lambda_{1}>0$ (the case $\lambda_{1}<0$ is entirely similar). It is then clear that the inviscid equation requires one boundary condition at $x_{1}=$ 0 and no boundary condition at $x_{1}=1$. Therefore, there will be a boundary layer occurring near $x_{1}=1$ and no boundary layer occurring near $x_{1}=0$. The boundary data $b_{0}\left(x^{\prime}, t\right), b_{1}\left(x^{\prime}, t\right)$ and initial data $u_{0}(x)$ all contribute to the boundary layer but in different ways: the boundary layer excited by $b_{1}\left(x^{\prime}, t\right)$ takes place immediately and is nontrivial as long as $b_{1}\left(x^{\prime}, t\right) \neq 0$. This is solely due to the discrepancy of the numbers of the boundary conditions at $x_{1}=1$; on the other hand, $u_{0}(x)$ and $b_{0}\left(x^{\prime}, t\right)$ have to depend on the transport process to contribute to the boundary layer at $x_{1}=1$. Accordingly, the boundary layer caused by $u_{0}(x)$ is nontrivial only in the time range $0<t<1 / \lambda_{1}$ and the boundary layer effect caused by $b_{0}\left(x^{\prime}, t\right)$ only shows up after $t=1 / \lambda_{1}$. As in the quarter space problem, these boundary layers are suppressed to the next order when the boundary condition at $x_{1}=1$ is non-Dirichlet, i.e., $B_{1} \neq 0$.

The validity of the viscosity limit and the corresponding boundary layers near $x_{1}=1$ can be justified by the same analysis used in the proof of Theorems 1.2-1.4. Instead of 
(9) and (10), we now have (assuming $\lambda_{1}>0$ )

$$
\begin{aligned}
& \int_{0}^{\infty} \int_{[0,1] \times \mathbf{R}^{n-1}}\left|u^{\varepsilon}(x, t)-u(x, t)\right|^{2} e^{-2 \alpha t} d x d t \\
& \quad \leq \begin{cases}O(1) \varepsilon\left\|b_{0}\right\|_{H^{2}}^{2}+O(1) \varepsilon\left\|b_{1}\right\|_{L^{2}}^{2}+O(1) \varepsilon\left\|u_{0}\right\|_{H^{2}}^{2} & B_{1}=0 \\
O(1) \varepsilon^{2}\left\|b_{0}\right\|_{H^{2}}^{2}+O(1) \varepsilon^{3}\left\|b_{1}\right\|_{L^{2}}^{2}+O(1) \varepsilon^{2}\left\|u_{0}\right\|_{H^{2}}^{2} & B_{1} \neq 0\end{cases}
\end{aligned}
$$

and

$$
\begin{aligned}
& \int_{0}^{\infty} \int_{[0,1] \times \mathbf{R}^{n-1}}\left|u^{\varepsilon}(x, t)-u(x, t)-u^{b . l .}(x, t)\right|^{2} e^{-2 \alpha t} d x d t \\
& \leq O(1) \varepsilon^{2}\left\|b_{0}\right\|_{H^{2}}^{2}+O(1) \varepsilon^{3}\left\|b_{1}\right\|_{H^{1}}^{2}+O(1) \varepsilon^{2}\left\|u_{0}\right\|_{H^{5}}^{2} .
\end{aligned}
$$

For $\lambda_{1}=0$, the boundary layers are of diffusion type and exist near both boundaries $x_{1}=0$ and $x_{1}=1$. No transport process is available in the present case; therefore, the boundary data $b_{0}\left(x^{\prime}, t\right)$ and $b_{1}\left(x^{\prime}, t\right)$ are now solely responsible for the boundary layers near $x_{1}=0$ and $x_{1}=1$ respectively. The corresponding convergence estimates are analogous to those in (9) and (10).

6.2. System Case. For systems of convection diffusion equations, the interaction of different waves can produce interesting new phenomena in the zero viscosity limit. In particular, through the coupling of boundary conditions (the amplification of the $\partial_{x} u^{\varepsilon}$ term in the boundary condition), the boundary layers in the outlet components of $u^{\varepsilon}$ can lead to catastrophic reflection waves dominating the inlet in the zero viscosity limit. However, for most boundary conditions, including Neumann boundary conditions (the coefficient matrix $B$ in (3) has full rank), and the Dirichlet boundary condition $(B=0)$, similar convergence results as in Theorems $1.2-1.4$ still hold. Details can be found in $[14]$.

\section{References}

[1] B. Engquist and A. Majda, Absorbing boundary conditions for the numerical simulation of waves, Math. Comp. 31 (1977), no. 139, 629-651.

[2] B. Engquist and A. Majda, Radiation boundary conditions for acoustic and elastic wave calculations, Comm. Pure Appl. Math. 32 (1979), no. 3, 314-358.

[3] M. Gisclon and D. Serre, Etude des conditions aux limites pour un système strictement hyperbolique, via l'approximation parabolique, (French) C. R. Acad. Sci. Paris, Série I Math., 319 (1994), no. 4, 377-382.

[4] M. Goldberg and E. Tadmor, Scheme-independent stability criteria for difference approximations of hyperbolic initial-boundary value problems, II., Math. Comp. 36 (1981), 603-626.

[5] E. Grenier and O. Guès, Boundary layers for viscous perturbations of noncharacteristic quasilinear hyperbolic problems, J. Differential Equations, 143 (1998), 110-146.

[6] M. J. Grote and J. B. Keller, Exact nonreflecting boundary condition for elastic waves, SIAM J. Appl. Math. 60 (2000), 803-819.

[7] B. Gustafsson, H.-O. Kreiss, and A. Sundström, Stability theory of difference approximations for mixed initial boundary value problems, II., Math. Comp. 26 (1972), 649-686.

[8] B. Gustafsson, H.-O. Kreiss, and J. Oliger, Time dependent problems and difference methods, Pure and Applied Mathematics. John Wiley \& Sons, Inc., New York, 1995.

[9] K. T. Joseph, Boundary layers in approximate solutions, Trans. Amer. Math. Soc. 314 (1989), 709-726.

[10] K. T. Joseph and P. G. LeFloch, Boundary layers in weak solutions of hyperbolic conservation laws, Arch. Ration. Mech. Anal. 147 (1999), no. 1, 47-88. 
[11] H.-O. Kreiss, Stability theory for difference approximations of mixed initial boundary value problems. I, Math. Comp. 22 (1968), 703-714.

[12] H.-O. Kreiss and J. Lorenz, Initial-boundary value problems and the Navier-Stokes Equations, Academic Press, New York, 1989.

[13] H.-O. Kreiss and L. Wu, Stable difference approximations for parabolic equations. Theory and numerical methods for initial-boundary value problems. Math. Comput. Modelling 20 (1994), no. 10-11, 123-143.

[14] H.-E. Lin, J.-G. Liu, and W.-Q. Xu, Effects of small viscosity and far-field boundary conditions for hyperbolic systems, Preprint.

[15] J.-G. Liu and Z. Xin. Boundary-layer behavior in the fluid-dynamic limit for a nonlinear model Boltzmann equation, Arch. Rational Mech. Anal. 135 (1996), no. 1, 61-105.

[16] D. Michelson, Convergence theorem for difference approximations for hyperbolic quasilinear initialboundary value problems, Math. Comp. 49 (1987), no. 180, 445-459.

[17] S. Osher, Stability of difference approximations of dissipative type for mixed initial-boundary value problems. I, Math. Comp. 23 (1969), 335-340.

[18] S. Osher, Systems of difference equations with general homogeneous boundary conditions, Trans. Amer. Math. Soc., 137 (1969), 177-201.

[19] Z. Xin and W.-Q. Xu, Stiff well-posedness and asymptotic convergence for a class of linear relaxation systems in a quarter plane. J. Differential Equations, 167 (2000), 388-437.

[20] W.-Q. Xu, Initial-boundary value problem for a class of linear relaxation systems in arbitrary space dimensions, J. Differential Equations, 183 (2002), 462-496.

[21] L. Ying and H. Han, The infinite element method for unbounded regions and inhomogeneous problems, (Chinese) Acta Math. Sinica 23 (1980), 118-127. 